Carlos I. Echeverría Arriagada, Universidad Estatal de Pensilvania

\title{
Sobre un error en la traducción española de "Principes de syntaxe fonctionnelle" de Coseriu
}

La importancia de la traducción en la ciencia resulta indudable si admitimos, con Montgomery (2000: ix), que "science exists because scientists are writers and speakers" ('la ciencia existe porque los científicos son escritores y hablantes') y que "there are no boundaries, no walls, between the doing of science and the communication of it" ("no existen límites, ni muros, entre el ejercicio de la ciencia y su comunicación'). Una de las consecuencias de reconocer la comunicación de los hallazgos científicos como parte inherente de la ciencia es que un error en una traducción científica, en caso de entorpecer la transmisión del conocimiento en la comunidad o cultura de llegada, ha de considerarse un error en la práctica científica misma. Por supuesto, esto es verdad también en la lingüística: un error en la traducción de un texto glotológico ha de tomarse como una amenaza a la propia integridad de la disciplina. Por ello, en esta nota nos hemos propuesto el modesto objetivo de llamar la atención de los estudiosos del lenguaje, en especial en el ámbito hispánico, sobre un error en la versión española de un artículo que constituye una pieza importantísima - aunque no suficientemente valorada ni divulgada - de la lingüística del siglo XX. Nos referimos a la traducción de "Principes de syntaxe fonctionnelle" de Coseriu (1989) realizada por Teresa Moure, la cual apareció hace ya casi tres décadas en el primer volumen de la revista Moenia (Coseriu 1995).

En dicho artículo, publicado originalmente en el vigesimoséptimo volumen de Travaux de linguistique et de philologie, Coseriu presenta, como se podía esperar por el título, los principios y las tareas centrales de la sintaxis o gramática funcional, cuyo cometido es, como traduce con acierto Moure, el de "establecer la estructura semánticogramatical propia de una lengua determinada" (Coseriu 1995: 11). Siendo esto así, uno de los aspectos centrales del texto $-\mathrm{y}$ en realidad de buena parte de la producción científica de Coseriu - es la distinción de varios tipos de significado y de distintos tipos de signos según los tipos de significado que expresan. En esta oportunidad nos interesan 
puntualmente los conceptos de significado léxico, significado categorial y pronombre o categorema, que en el texto original aparecen explicados en el siguiente pasaje:

Le signifié lexical, c'est le signifié qui correspond d'une façon immédiate à l'organisation du monde extralinguistique au moyen des langues et il représente par là, pour le dire avec Husserl (Erfahrung und Urteil), la substance, le «Was der Erfassung », le type de réalité nommé par le langage. C'est, par exemple, le signifié qu'ont en commun les mots de la série blanc - blancheur blanchir (et, si l'on veut, blanchement). Dans tous ces mots, il s'agit du même type de couleur, type différent des types de nommés par les séries vert - verdeur - verdir, rouge - rougeur - rougir etc. Le signifié catégoriel, c'est le signifié que les termes blanc - vert - rouge, blancheur - verdeur rougeur, blanchir - verdir - rougir des ces séries ont chaque fois en commun et qui, par contre, est différent pour les termes de chaque série ; il ne concerne pas la substance mais la forme de l'intuition de la réalité, correspondant, toujours selon Husserl, à des différences in der Weise der Erfassung. Ainsi, dans une série telle que blanc - blancheur - blanchir, la substance de l'intuition est la même mais elle est conçue et présentée chaque fois sous une forme différente. Il n'y a dans les langues que quatre types de signifiés catégoriels possibles : substantif, adjectif, verbe, adverbe ; mais entre eux il peut y avoir fusion (par exemple : verbe + adjectif, adjectif + adverbe) et ils peuvent, d'autre part, présenter des sous-divisions. Les pronoms (ou " catégorèmes ») sont à cet égard des « formes sans substance » : ils présentent uniquement ou en premier lieu un signifié catégoriel (ils sont substantifs, adjectifs, adverbes, parfois même verbes), n'ayant pourtant pas de signifié lexical ou n'ayant qu'un signifié lexical générique ( « personne », « chose », « lieu », « temps », « modalité », « activité » etc.) (Coseriu 1989: 20).

Centrémonos ahora en la última parte de este pasaje, concerniente al concepto de pronombre o categorema, parte que en la versión española se traduce del siguiente modo:

Los pronombres (o categoremas) son en esta perspectiva "formas sin sustancia": presentan únicamente o en primer lugar un significado categorial (son sustantivos, adjetivos, adverbios, a veces incluso verbos) que, sin embargo, no tienen significado léxico o no tienen un significado léxico genérico ("persona", "cosa", "lugar", "tiempo", "modalidad", "actividad”, etc.) (Coseriu 1995: 2425).

A primera vista podrá parecer que la traducción es, en lo esencial, fiel al original; sin embargo, si se presta suficiente atención a ambos textos se podrá notar que la versión española contiene un error de no menor importancia (fuera de que, después del segundo paréntesis, se recurra a una oración de relativo como si el paréntesis no se hubiera cerrado). Nos referimos a la frase no tienen un significado léxico genérico, que corresponde a la frase n'ayant qu'un signifié lexical générique del texto original. En efecto, la frase francesa no 
quiere decir lo que dice la traducción, sino más bien lo contrario: que los pronombres pueden tener un significado léxico genérico, sentido que en el artículo original viene determinado por $q u$ ' antes de la frase nominal. Obsérvese, a modo de comparación, que este sentido aparece con claridad también en la versión rumana del mismo artículo, de responsabilidad de Emma Tămâianu:

Sub acest aspect, pronumele (,,categoremele”) sunt „forme fără substanță”: ele prezintă exclusiv sau în primul rând un semnificat categorial (sunt substantive, adjective, adverbe, uneori chiar verbe), neavând însă un semnificat lexical sau având numai unul generic (,persoană”, „lucru”, „loc”, „,timp”, ,modalitate”, ,,activitate” etc.) (Coseriu 1994-1995: 44).

El error que hemos señalado, aunque grave a nuestro parecer, no resulta sorprendente si se tiene en cuenta que, en casi todos los demás trabajos de Coseriu en que se habla con algún detalle de estos tipos de significado (entendidos como tipos de contenido de lengua) y de los tipos de signos correspondientes (p. ej., Coseriu 1955-1956, 1977, 1978, 1981, 1982, 2016), el autor considera los categoremas como palabras que, teniendo significado categorial, sencillamente no poseen significado léxico, sin referencia a la posibilidad de un significado léxico "genérico". Así, por ejemplo, en su conocido ensayo "Determinación y entorno" Coseriu escribe que "los pronombres sustantivos sólo pueden denotar gracias a la situación” ya que "tienen significado categorial (son 'sustantivos'), pero no tienen significado léxico" (1955-1956: 46), tras lo cual define los categoremas como "palabras que tienen sólo significado categorial” (óp. cit.: 52). Y en La semántica en la lingüística del siglo $X X$, libro de reciente aparición cuyo contenido corresponde a un curso impartido en 1998, leemos que los pronombres "solo tienen significado categorial" y "no tienen significado léxico"; que "son solo formas de lo aprehendido, sin el qué de lo aprehendido" (2016: 70). La única excepción que conocemos a este respecto, aparte del mentado artículo de 1989, es la nota "Tesis acerca del 'significado", donde Coseriu nos dice que "los pronombres y los numerales tienen sólo significado categorial, y no tienen 
significado léxico (específico)" (1998: 85), siendo el último paréntesis la única indicación de que estos signos pueden tener algún significado léxico estable, aunque sea "genérico"1.

De este modo, "Principes de syntaxe fonctionnelle" representa un importante cambio en la tipología de signos de Coseriu al que nadie parece haberse referido hasta ahora, aun si se trata de una modificación a la que el lingüista rumano no se adhirió de manera consistente. Este cambio, a nuestro parecer, acerca a Coseriu a lingüistas como Alarcos Llorach, por mencionar un nombre conocido en el ámbito hispánico, quien en su caracterización de los pronombres demostrativos españoles reconoce, contra la concepción popular, que estos no solo poseen un significado estable, sino que además poseen un significado propiamente léxico, que el estudioso español llama "el lexema del demostrativo" (1977: 55). La definición de los pronombres que encontramos en el texto coseriano de 1989 resulta entonces interesante tanto desde el punto de vista teórico como desde el punto de vista historiográfico, y esto es algo que resulta imposible apreciar si solo se tiene acceso a la versión española, la cual en las últimas décadas varios en el ámbito hispánico hemos citado sin ninguna observación a este respecto y sin referencia al texto original (vid., p. ej., Álvarez Castro 2005, 2006; Arrizabalaga 2011; Echeverría Arriagada 2016; García González 2017; Gutiérrez Rodríguez 2016; Mancera Rueda 2008, 2009; San Julián Solana 2016).

Todo lo anterior hace necesario a nuestro juicio llamar la atención sobre el error al que nos hemos referido, el cual, en todo caso, no logra quitar mérito a la labor de Moure, quien en general logra transmitir las ideas de Coseriu de manera elogiable. Esperamos, pues, que esta breve nota sirva para alertar a los lingüistas en el ámbito hispánico sobre este lapsus traduccional, así como para motivar nuevas reflexiones sobre la tipología de signos coseriana. Algunas de las preguntas que cabe plantearse en esta materia son las siguientes: ¿Se justifica realmente una distinción tajante entre palabras lexemáticas y categoremáticas,

1 En su trabajo inédito titulado Teoría del nombre propio, el lingüista rumano escribe que los categoremas "se refieren siempre a un significado léxico dado por la situación o por otras palabras del contexto" (Coseriu, s. f., § 2.3.2, n. 13). Mas debe observarse que aquí significado no tiene exactamente el mismo sentido que en otros trabajos de Coseriu, donde el término se reserva solo para el "contenido dado por la lengua empleada en el discurso y exclusivamente por ella, es decir, la organización de las posibilidades de designación específicas de una lengua dada" (Coseriu 1995: 11), para citar la traducción de Moure. 
o conviene más bien pensar en diversos grados de especificidad léxica? ¿Existen siquiera palabras no puramente morfemáticas que no conformen ninguna sustancia semántica más allá de la correspondiente a sus accidentes gramaticales? Y, aun si aceptamos la posibilidad de signos no gramaticales de denotación realmente infinita, aplicables a absolutamente cualquier entidad o estado de cosas, ¿resulta pertinente dar por sentado que se trata de unidades sin significado léxico, o convendría quizá hablar de una forma especial de significado léxico? Desde luego, estos interrogantes tendrán que quedar para otra oportunidad. 


\section{Bibliografía}

Alarcos Llorach, Emilio (1977): “Los demostrativos en español”, en: Verba 31, 53-63.

Álvarez Castro, Natalia (2005): “Tradición y novedad del entorno universo de discurso de Eugenio Coseriu", en: Antonio Roldán Pérez, Ricardo Escavy Zamora, E. Hernández Sánchez, José Miguel Hernández Terrés y M.a Isabel López Martínez (eds.): Caminos actuales de la historiografía lingüística: Actas del V Congreso Internacional de la Sociedad Española de Historiografía Lingüística, I. Murcia: Universidad de Murcia, 223-233.

— (2006): “Consideraciones metalingüísticas sobre el entorno 'universo del discurso' de Eugenio Coseriu”, en: Ramón González Ruiz y Manuel Casado Velarde (eds.): Discurso, lengua y metalenguaje: balance y perspectivas. Hamburgo: Helmut Buske Verlag, 21-35.

Arrizabalaga, Carlos (2011): "Ya con Felicitas fuimos a buscar al párroco de Santa Catalina'. Discordancia oracional y gramaticalización en el español peruano”, en: Lexis 35, 1, 163-186.

Coseriu, Eugenio (1955-1956): “Determinación y entorno: dos problemas de una lingüística del hablar", en: Romanistisches Jahrbuch 7, 29-54.

- (1977): “La 'situación' en la lingüística", en: El hombre y su lenguaje: estudios de teoría y metodología lingüística. Madrid: Gredos, 240-256.

- (1978): "Semántica y gramática”, en: Gramática, semántica, universales: estudios de lingüística funcional. Madrid: Gredos, 128-147.

- (1981): "Introducción al estudio estructural del léxico", en: Principios de semántica estructural. Madrid: Gredos, 87-142.

- (1989): "Principes de syntaxe fonctionnelle", en: Travaux de linguistique et de philologie 27, 5-46.

— (1994-1995): “Principii de sintaxă funcțională”, en: Dacoromania 1, 1-2, 29-68.

— (1995): "Principios de sintaxis funcional", en: Moenia 1, 11-46.

— (1998): “Tesis acerca del 'significado", en: Lexis 21, 2, 83-86. 
- (2016): La semántica en la lingüística del siglo XX: tendencias y escuelas. Madrid: Arco/Libros.

- (s. f.): Teoría del nombre propio (texto inédito).

Echeverría Arriagada, Carlos I. (2016): "Criterios estilísticos y funcionalidad: a propósito del concepto de simetría compositiva", Hápax 9, 127-139. Disponible en http://www.revistahapax.es/pages/numeros $16 . h t m l$

García González, Cristina (2017): Visión panorámica del funcionalismo español. Tesis doctoral, Universidad de León. Disponible en https://buleria.unileon.es/handle/10612/6779

Gutiérrez Rodríguez, Edita (2016): “Gramática estructural”, en: Javier Gutiérrez-Rexach (ed.): Enciclopedia de Lingüistica Hispánica, 1. Londres: Routledge, 113-126.

Mancera Rueda, Ana. (2008): "Rasgos de sintaxis oral-coloquializada en los diálogos de los siglos XVI y XVII destinados a la enseñanza de español a extranjeros”, en: Boletín de la Real Academia Española 88, 298, 229-258.

- (2009): 'Oralización' de la prensa española: la columna periodística. Berna: Peter Lang.

Montgomery, Scott L. (2000): Science in translation. Chicago: University of Chicago Press.

San Julián Solana, Javier (2016): "El sustantivo cuantificador en la lingüística hispánica: revisión crítica y nueva propuesta", en: Círculo de Lingüistica Aplicada a la Comunicación 68, 380-435. 